Nervenarzt 2022 $93: 305-308$

https://doi.org/10.1007/s00115-021-01148-3

Angenommen: 20. März 2021

Online publiziert: 17. Juni 2021

๑ Der/die Autor(en) 2021

\author{
Hanna Reich ${ }^{1,2} \cdot$ Andreas Czaplicki $^{2}$ Christian Gravert ${ }^{3}$. Ulrich Heger| ${ }^{2,4,5}$ \\ ${ }^{1}$ Forschungszentrum Depression der Stiftung Deutsche Depressionshilfe, Klinik für Psychiatrie, \\ Psychosomatik und Psychotherapie, Johann-Wolfgang-Goethe-Universität, Frankfurt am Main, \\ Deutschland \\ ${ }^{2}$ Stiftung Deutsche Depressionshilfe, Leipzig, Deutschland \\ ${ }^{3}$ Deutsche Bahn AG, Berlin, Deutschland \\ ${ }^{4}$ Johann Christian Senckenberg Distinguished Professorship, Klinik für Psychiatrie, Psychosomatik und \\ Psychotherapie, Johann-Wolfgang-Goethe-Universität, Frankfurt am Main, Deutschland \\ ${ }^{5}$ Universitätsklinikum Frankfurt, Klinik für Psychiatrie, Psychosomatik und Psychotherapie, Stiftung \\ Deutsche Depressionshilfe, Frankfurt am Main, Deutschland
}

\title{
Negative Effekte der COVID-19- Maßnahmen auf die Versorgung depressiv Erkrankter
}

\section{Ergebnisse einer repräsentativen Bevölkerungsbefragung}

Akteure in der Politik, im Medizinbetrieb und in vielen anderen Bereichen haben eingreifend und teils rasch und zielgerichtet auf die Bedrohungen durch die COVID-19-Pandemie reagiert. Mit Blick auf die Situation in anderen Ländern wurden die getroffenen Maßnahmen meist als erfolgreich, wenn nicht gar vorbildlich dargestellt und dabei auf die Infektionszahlen und COVID-19assoziierten Todesfälle verwiesen. Diese Zahlen allein sind jedoch für eine Erfolgsbeurteilung nicht ausreichend, da dadurch die „Nebenwirkungen“ der getroffenen Maßnahmen vernachlässigt werden. Entscheidend ist die Frage nach dem Verhältnis zwischen dem Ausmaß an Leid und Tod, das durch die getroffenen Maßnahmen einerseits verhindert und andererseits verursacht wird. Berichte über gravierende negative gesundheitliche Folgen dieser Maßnahmen häufen sich. In Deutschland wie auch in anderen Ländern wurde nach Ausbruch der Corona-Pandemie über eine verzögerte medizinische Kontaktaufnahme von Patient:innen nach Herzinfarkt oder Schlaganfall verbunden mit deutlich erhöhtem intrahospitalem Sterberisiko berichtet $[2,8,9]$. Auch die Versorgung von Patient:innen mit
Suchterkrankungen war bedingt durch monatelange Schließungen ambulanter Drogenhilfeeinrichtungen erschwert [4].

Mit dieser Arbeit soll ein Beitrag zu der dringend benötigten Diskussion der Kosten-Nutzen-Abschätzung bzgl. der Maßnahmen zur Eindämmung des Infektionsgeschehens geleistet werden, indem aus Betroffenensicht deren Einfluss auf die medizinische und psychotherapeutische Versorgung depressiv Erkrankter dargestellt wird.

In einer bevölkerungsrepräsentativen Umfrage der deutschen Wohnbevölkerung im Alter von 18 bis 69 Jahren wurden 5178 Personen $(n=2660$ weiblich) im Zeitraum vom 26.06 .2020 bis 08.07.2020 durch das zertifizierte Befragungsunternehmen (ISO 26362) Respondi AG online befragt (Teilnahmequote: $31,3 \%$ ). Die Stichprobe stellt eine mehrfach geschichtete Quotenauswahl anhand der verschränkten Merkmale Geschlecht, Alter, Bundesland/Bundesländergruppe entsprechend der aktuellen Bevölkerungsfortschreibungen des Statistischen Bundesamts dar. Alle Teilnehmenden waren im Access Panel registriert und haben ihre schriftliche Einwilligungserklärung zur Teilnahme gegeben. Eine Aufwandsentschädigung erfolgte in
Form von Punkten (Gegenwert: $1 €$ ), die für Internetkäufe eingesetzt werden können. Einschränkungen in der allgemeinen medizinischen Versorgung wurden für den Befragungszeitraum („aktuell“) und retrospektiv für die erste Lockdownphase („4 Wochen des Lockdowns“) folgendermaßen erfragt: „Durch die Maßnahmen gegen Corona sind bei mir oder einem nahen Angehörigen wichtige Arzttermine ausgefallen/wichtige medizinische Behandlungen nicht durchgeführt worden/die Behandlungsmöglichkeiten beeinträchtigt gewesen " (LikertSkala 1-4). Teilnehmende mit diagnostizierter Depression $(n=1,094$, Ja, bei mir ist bereits einmal die Diagnose Depression gestellt worden“; davon $n=197$ „Ich befinde mich aktuell in einer depressiven Phase") wurden zu spezifischen Auswirkungen auf die Versorgung ihrer Depressionserkrankung befragt ( $\mathrm{ja} /$ nein), die Datenauswertung erfolgte mit Stata SE15.1 $(\alpha=0,05)$.

Mehr als ein Drittel der befragten Allgemeinbevölkerung gab an, während des ersten Lockdowns Einschränkungen der medizinischen Versorgung bei sich selbst oder einem nahen Angehörigen erlebt zu haben; auch zum Befragungszeitpunkt im Sommer berichtete dies immer noch 


\section{Kurzbeiträge}

Tab. 1 Einschränkungen in der allgemeinen medizinischen Versorgung während der COVID-19-Pandemie („bei mir oder einem nahen Angehörigen“, Selbstbericht)

\begin{tabular}{|c|c|c|c|c|c|c|c|c|c|c|}
\hline & & \multicolumn{4}{|c|}{ Während des 1. Lockdowns } & \multicolumn{4}{|c|}{ Aktuell (Juni/Juli 2020) } & \multirow{2}{*}{$\begin{array}{l}\text { Signifikanz } \\
\text { Zeiteffekt }\end{array}$} \\
\hline & & $n$ & $\% w$ & {$[95 \%$} & & $n$ & $\% w$ & {$[95 \%$} & & \\
\hline \multirow{2}{*}{$\begin{array}{l}\text { Lebenszeit- } \\
\text { diagnose De- } \\
\text { pression }\end{array}$} & $\begin{array}{l}\text { Aktuelle depressive Episode } \\
(n=197)\end{array}$ & 110 & 56,3 & 49,2 & 63,0 & 83 & 42,4 & 35,7 & 49,5 & \\
\hline & In Remission $(n=897)$ & 352 & 39,4 & 36,2 & 42,6 & 221 & 24,7 & 22,0 & 27,7 & $p<0,001$ \\
\hline \multicolumn{2}{|c|}{ Allgemeinbevölkerung $(n=4084)$} & 1413 & 34,8 & 33,3 & 36,3 & 889 & 22,0 & 20,7 & 23,3 & $p<$ \\
\hline \multicolumn{2}{|c|}{ Signifikanz Gruppeneffekt } & - & $p<0,001$ & - & - & - & $p=0,004$ & - & - & - \\
\hline \multicolumn{11}{|c|}{$\begin{array}{l}\text { Nach Alter, Geschlecht und Bundesland gewichtete Prozentangaben (\%w [95\%iges Konfidenzintervall]) } \\
\text { Die Antwort „trifft zu“ oder „trifft sehr zu“ auf mindestens eins der drei Items („Durch die Maßnahmen gegen Corona sind bei mir oder einem nahen Ange- } \\
\text { hörigen wichtige Arzttermine ausgefallen/wichtige medizinische Behandlungen nicht durchgeführt worden/die Behandlungsmöglichkeiten beeinträchtigt } \\
\text { gewesen") wurde als Einschränkung gewertet } \\
\text { Test der Gruppeneffekte mit univariaten ANOVAs, Test der Zeiteffekte mittels gepaarter t-Tests } \\
\text { Signifikante Unterschiede sind fett hervorgehoben }\end{array}$} \\
\hline
\end{tabular}

Tab. 2 Terminausfälle in derVersorgung depressiver Erkrankungen (Selbstbericht, $n=1094$ Personen mit diagnostizierten depressiven Erkrankungen) während der COVID-19-Pandemie nach Versorgungsform, Geschlecht und Alter

\begin{tabular}{|c|c|c|c|c|c|c|c|c|c|c|}
\hline \multirow{2}{*}{$\begin{array}{l}\begin{array}{l}\text { Ausfall von } \\
\text { Terminen }\end{array} \\
\text { Hausarzt }\end{array}$} & \multirow[b]{2}{*}{-} & \multirow[b]{2}{*}{-} & \multirow{2}{*}{$\begin{array}{l}\text { n/Gesamt } \\
112 / 1094\end{array}$} & \multirow{2}{*}{$\begin{array}{l}\% w \\
10,3\end{array}$} & \multicolumn{2}{|c|}{ [95\%-Cl] } & \multirow{2}{*}{$\begin{array}{l}\text { OR } \\
-\end{array}$} & \multicolumn{2}{|c|}{ [95\%-Cl] } & \\
\hline & & & & & 8,6 & 12,2 & & - & - & \\
\hline & Geschlecht & W (= Ref.) & $59 / 639$ & 9,2 & 7,2 & 11,7 & 1,00 & - & - & - \\
\hline & & M & $53 / 455$ & 11,7 & 9,0 & 15,0 & 1,30 & 0,88 & 1,93 & 0,193 \\
\hline & Alter & 18-29 J & $10 / 135$ & 7,7 & 4,1 & 13,8 & 0,72 & 0,36 & 1,43 & 0,344 \\
\hline & & 30-59 J. (= Ref.) & $76 / 723$ & 10,5 & 8,5 & 13,0 & 1,00 & - & - & - \\
\hline & & 60-69J & $26 / 236$ & 11,0 & 7,6 & 15,7 & 1,07 & 0,66 & 1,71 & 0,790 \\
\hline \multirow[t]{6}{*}{ Facharzt } & - & - & $171 / 1094$ & 15,8 & 13,8 & 18,2 & - & - & - & - \\
\hline & Geschlecht & W (=Ref.) & $78 / 639$ & 12,2 & 9,9 & 15,0 & 1,00 & - & - & - \\
\hline & & M & $93 / 455$ & 20,7 & 17,2 & 24,7 & 1,89 & 1,36 & 2,64 & $<0,001$ \\
\hline & Alter & 18-29 J & $24 / 135$ & 18,6 & 12,7 & 26,3 & 1,28 & 0,78 & 2,08 & 0,324 \\
\hline & & 30-59 J. (= Ref.) & $113 / 723$ & 15,7 & 13,2 & 18,6 & 1,00 & - & - & - \\
\hline & & 60-69J & $34 / 236$ & 14,4 & 10,5 & 19,6 & 0,94 & 0,62 & 1,43 & 0,764 \\
\hline \multirow[t]{6}{*}{ Psychotherapeut } & - & - & $134 / 1094$ & 12,4 & 10,5 & 14,5 & - & - & - & - \\
\hline & Geschlecht & W (= Ref.) & $74 / 639$ & 11,6 & 9,3 & 14,3 & 1,00 & - & - & - \\
\hline & & M & $60 / 455$ & 13,3 & 10,5 & 16,8 & 1,17 & 0,81 & 1,70 & 0,396 \\
\hline & Alter & 18-29 J & $26 / 135$ & 19,4 & 13,5 & 27,1 & 1,65 & 1,02 & 2,68 & 0,042 \\
\hline & & 30-59 J. (= Ref.) & $93 / 723$ & 12,8 & 10,6 & 15,5 & 1,00 & - & - & - \\
\hline & & 60-69J & $15 / 236$ & 6,4 & 3,8 & 10,3 & 0,46 & 0,26 & 0,82 & 0,008 \\
\hline \multirow{6}{*}{$\begin{array}{l}\text { Geplanter statio- } \\
\text { närer Aufenthalt }\end{array}$} & - & - & $35 / 1094$ & 3,2 & 2,3 & 4,5 & - & - & - & - \\
\hline & Geschlecht & W (= Ref.) & $19 / 639$ & 3,0 & 1,9 & 4,7 & 1,00 & - & - & - \\
\hline & & M & $16 / 455$ & 3,5 & 2,2 & 5,7 & 1,15 & 0,59 & 2,27 & 0,680 \\
\hline & Alter & 18-29 J & $5 / 135$ & 3,7 & 1,5 & 8,8 & 0,97 & 0,37 & 2,55 & 0,947 \\
\hline & & 30-59 J. (= Ref.) & $28 / 723$ & 3,9 & 2,7 & 5,6 & 1,00 & - & - & - \\
\hline & & $60-69 \mathrm{~J}$ & $2 / 236$ & 0,9 & 0,2 & 3,4 & 0,21 & 0,05 & 0,91 & 0,037 \\
\hline \multirow[t]{6}{*}{ Selbsthilfegruppe } & - & - & $67 / 1094$ & 6,2 & 4,9 & 7,8 & - & - & - & - \\
\hline & Geschlecht & W (=Ref.) & $41 / 639$ & 6,4 & 4,7 & 8,6 & 1,00 & - & - & - \\
\hline & & M & $26 / 455$ & 5,9 & 4,0 & 8,5 & 0,88 & 0,53 & 1,47 & 0,634 \\
\hline & Alter & 18-29 J & $6 / 135$ & 4,4 & 2,0 & 9,6 & 0,58 & 0,24 & 1,38 & 0,214 \\
\hline & & 30-59 J. (= Ref.) & $53 / 723$ & 7,4 & 5,7 & 9,5 & 1,00 & - & - & - \\
\hline & & $60-69 \mathrm{~J}$ & $8 / 236$ & 3,4 & 1,7 & 6,6 & 0,43 & 0,20 & 0,92 & 0,030 \\
\hline
\end{tabular}

Nach Alter, Geschlecht und Bundesland gewichtete Prozentangaben (\%w [95\%iges Konfidenzintervall]) und Odds Ratios (OR) aus multiplen logistischen Regressionen (für Geschlecht und Alter adjustiert)

Von $n=1094$ Personen mit diagnostizierten depressiven Erkrankungen waren $n=197$ aktuell depressiv, $n=897$ remittiert depressiv

Signifikante Unterschiede sind fett hervorgehoben

Die Referenzkategorien sind mit (= Ref.) gekennzeichnet 
fast ein Viertel aller Befragten (• Tab. 1). Unter den Teilnehmenden mit einer aktuellen depressiven Krankheitsphase lagen die berichteten Einschränkungen mit $56 \%$ (Lockdown) bzw. $42 \%$ (Juni/Juli) nochmals höher. Hierbei gaben außerdem $13 \%$ der Betroffenen an, selbst ambulante Termine abgesagt zu haben, weil ihnen die Situation zu unsicher gewesen sei. Jeder fünfte männliche Teilnehmer mit diagnostizierter Depression berichtete von Terminausfällen beim Facharzt, das Risiko für Ausfälle war damit fast doppelt so hoch wie bei Frauen (• Tab. 2). In Bezug auf psychotherapeutische Behandlungen war das Risiko von Terminausfällen für die Geschlechter vergleichbar, jedoch berichteten jüngere Erwachsene mit einer 1,6-fach erhöhten Wahrscheinlichkeit von Ausfällen psychotherapeutischer Termine im Vergleich zu Erwachsenen mittleren Alters. Dass über 60-Jährigen seltener über Ausfälle von Psychotherapiesitzungen oder Selbsthilfegruppen berichten, mag Ausdruck dessen sein, dass derartige Versorgungsangebote älteren Menschen nur selten gemacht werden.

Hochgerechnet betrafen die hier erfragten Einschränkungen in der medizinischen Versorgung im ersten Jahr der Pandemie ca. 2 bis 3 Mio. Menschen mit affektiven Erkrankungen [3]. Diesen unter einer schweren, oft auch lebensbedrohlichen Erkrankung leidenden Menschen wurde eine schlechtere Versorgung ihrer Erkrankung zugemutet, um das Infektionsgeschehen zu verlangsamen oder Versorgungskapazitäten für COVID-19-Infizierte zu schaffen. Ähnliche Befunde zu einem Rückgang der Versorgung psychischer Erkrankungen liegen auch aus Großbritannien vor [5]. Männliche Befragungsteilnehmer und jüngere Erwachsene berichteten in der vorliegenden Befragung von stärkeren Einschränkungen als die jeweiligen Referenzgruppen (• Tab. 2). Gleichzeitig zeigte eine longitudinale Studie, dass das Stresserleben bei jungen Erwachsenen sowie depressive und Angstsymptome bei jungen Frauen im Besonderen im Zuge der Pandemie zunahmen [7]. Junge Erwachsene waren damit sowohl mit erhöhten Belastungen ihrer psychischen Gesundheit als auch mit stärkeren
Einschränkungen in der psychotherapeutischen Versorgung während der COVID-19-Pandemie konfrontiert. Eine mangelhafte Versorgung depressiver Erkrankungen junger Erwachsener kann mit hohen, langfristigen Kosten für Individuum und Gesellschaft einhergehen [1]. Die Sicherstellung zeitnaher, leitliniengerechter Behandlungen depressiver Erkrankungen sollte daher auch in Anbetracht ihres gesamtgesellschaftlichen Nutzens in den aktuellen politischen Entscheidungen stärker Eingang finden.

Bei der vorliegenden Arbeit handelt es sich um Analysen von Daten einer sozialwissenschaftlichen Befragung. Eine methodische Einschränkung betrifft Fragen, die sich auf den ersten Lockdown beziehen, da diese retrospektiv im Juni/Juli gestellt wurden. Zudem erfolgte bei der Erfragung allgemeiner Einschränkungen (• Tab. 1) keine Differenzierung zwischen Erkrankten selbst und Angehörigen; eine getrennte Auswertung ist an dieser Stelle somit nicht möglich.

Eine nicht nur auf das Infektionsgeschehen verengte Sicht macht deutlich, dass die Implementierung besonders strenger Maßnahmen in der COVID19-Pandemie nicht „Auf Nummer sicher gehen" bedeutet und dass es auch nicht um Gesundheit vs. Ökonomie geht, sondern um die richtige Balance zwischen Nutzen und Risiken der Maßnahmen. Eine Gesamtbilanzierung sollte weitere Aspekte beinhalten, die hier nicht betrachtet wurden. Diese umfassen neben ökonomischen und freiheitsrechtlichen Aspekten auch die Frage, ob sich die in einigen Bevölkerungsgruppen beobachteten Zunahmen von depressiven und Angstsymptomen [7] sowie des Alkoholkonsums [6] langfristig in einer Veränderung von Prävalenzraten psychischer Erkrankungen abbilden. Telemedizinische und digitale Behandlungsangebote haben während der Pandemie noch einmal an Bedeutung gewonnen. Einstellungen zu und Nutzung von diesen Angeboten sollten in zukünftigen Studien untersucht werden.

\section{Fazit für die Praxis}

Die Maßnahmen zur Verlangsamung
des COVID-19-Infektionsgeschehens gingen für depressiv erkrankte Menschen mit einem Verlust an Versorgungsqualität (ausgefallene ambulante Behandlungstermine, abgesagte stationäre Behandlungen) einher.

- Bei jungen und bei männlichen Erwachsenen war die Rate ausgefallener ambulanter Termine besonders hoch.

- Auch während der COVID-19-Pandemie bleibt das Recht von Patient: innen auf eine leitlinienkonforme Behandlung ihrer depressiven Erkrankung unverändert bestehen.

\section{Korrespondenzadresse}

\section{Prof. Dr. Ulrich Hegerl}

Universitätsklinikum Frankfurt, Klinik

für Psychiatrie, Psychosomatik und

Psychotherapie, Stiftung Deutsche

Depressionshilfe

Heinrich-Hoffmann-Str. 10, 60528 Frankfurt am

Main, Deutschland

ulrich.hegerl@kgu.de

Funding. Open Access funding enabled and organized by Projekt DEAL.

Interessenkonflikt. H. Reich de Paredes, A. Czaplicki, C. Gravert und U. Hegerl geben an, dass kein Interessenkonflikt besteht.

Open Access. Dieser Artikel wird unter der Creative Commons Namensnennung 4.0 International Lizenz veröffentlicht, welche die Nutzung, Vervielfältigung, Bearbeitung, Verbreitung und Wiedergabe in jeglichem Medium und Format erlaubt, sofern Sie den/die ursprünglichen Autor(en) und die Quelle ordnungsgemäß nennen, einen Link zur Creative Commons Lizenz beifügen und angeben, ob Änderungen vorgenommen wurden.

Die in diesem Artikel enthaltenen Bilder und sonstiges Drittmaterial unterliegen ebenfalls der genannten Creative Commons Lizenz, sofern sich aus der Abbildungslegende nichts anderes ergibt. Sofern das betreffende Material nicht unter der genannten Creative Commons Lizenz steht und die betreffende Handlung nicht nach gesetzlichen Vorschriften erlaubt ist, ist für die oben aufgeführten Weiterverwendungen des Materials die Einwilligung des jeweiligen Rechteinhabers einzuholen.

Weitere Details zur Lizenz entnehmen Sie bitte der Lizenzinformation auf http://creativecommons.org/ licenses/by/4.0/deed.de.

\section{Literatur}

1. Asselmann E, Wittchen HU, Lieb R, Beesdo-Baum K (2018) Sociodemographic, clinical, and functional long-term outcomes in adolescents and young 


\section{Kurzbeiträge}

adults with mental disorders. Acta Psychiatr Scand 137:6-17.https://doi.org/10.1111/acps.12792

2. Gluckman TJ, Wilson MA, Chiu ST et al (2020) Case rates, treatment approaches, and outcomes in acute myocardial infarction during the Coronavirus disease 2019 pandemic. JAMA Cardiol 5:1419-1424. https://doi.org/10.1001/ jamacardio.2020.3629

3. Jacobi F, Höfler M, Strehle J et al (2014) Psychische Störungen in der Allgemeinbevölkerung: Studie zur Gesundheit Erwachsener in Deutschland und ihr Zusatzmodul Psychische Gesundheit (DEGS1$\mathrm{MH})$. Nervenarzt 85:77-87

4. Krings A, Steffen G, Germershausen C, Zimmermann R (2020) Auswirkungen der COVID-19-Krise aufPräventionsangebote zu durch Blut und sexuell übertragenen Infektionen bei Drogengebrauchenden. Epidemiol Bull 42:3-9. https://doi.org/ 10.25646/7155

5. Mansfield KE, Mathur R, Tazare J et al (2021) Indirect acute effects of the COVID-19 pandemic on physical and mental health in the UK: a populationbased study. Lancet Digit Health. https://doi.org/ 10.1016/S2589-7500(21)00017-0

6. Manthey J, Kilian C, Schomerus G et al (2020) Alkoholkonsum in Deutschland und Europa während der SARS-CoV-2 Pandemie. Sucht 66:247-258. https://doi.org/10.1024/0939-5911/ a000686

7. Peters A, Rospleszcz S, Greiser KH et al (2020) The impact of the COVID-19 pandemic on self-reported health. Dtsch Arztebl Int 117:861-867. https://doi. org/10.3238/arztebl.2020.0861

8. Seiffert M, Brunner FJ, Remmel M et al (2020) Temporal trends in the presentation of cardiovascular and cerebrovascular emergencies during the COVID-19 pandemic in Germany: an analysis of health insurance claims. Clin Res Cardiol 109:1540-1548. https://doi.org/10.1007/s00392020-01723-9

9. Slagman A, Behringer W, Greiner F et al (2020) Medical emergencies during the COVID-19 pandemic - an analysis of emergency department data in Germany. Dtsch Arztebl Int 117:545-552. https://doi.org/10.3238/arztebl.2020.00545

\section{Hier steht eine Anzeige.}

\section{Springer}

